\title{
The agents-are-substitutes property in continuous generalized assignment problems
}

Citation for published version (APA):

Lok, R. B., Romero Morales, D., \& Vermeulen, A. J. (2005). The agents-are-substitutes property in continuous generalized assignment problems. METEOR, Maastricht University School of Business and Economics. METEOR Research Memorandum No. 009 https://doi.org/10.26481/umamet.2005009

Document status and date:

Published: 01/01/2005

DOI:

10.26481/umamet.2005009

Document Version:

Publisher's PDF, also known as Version of record

\section{Please check the document version of this publication:}

- A submitted manuscript is the version of the article upon submission and before peer-review. There can be important differences between the submitted version and the official published version of record.

People interested in the research are advised to contact the author for the final version of the publication, or visit the DOI to the publisher's website.

- The final author version and the galley proof are versions of the publication after peer review.

- The final published version features the final layout of the paper including the volume, issue and page numbers.

Link to publication

\footnotetext{
General rights rights.

- You may freely distribute the URL identifying the publication in the public portal. please follow below link for the End User Agreement:

www.umlib.nl/taverne-license

Take down policy

If you believe that this document breaches copyright please contact us at:

repository@maastrichtuniversity.nl

providing details and we will investigate your claim.
}

Copyright and moral rights for the publications made accessible in the public portal are retained by the authors and/or other copyright owners and it is a condition of accessing publications that users recognise and abide by the legal requirements associated with these

- Users may download and print one copy of any publication from the public portal for the purpose of private study or research.

- You may not further distribute the material or use it for any profit-making activity or commercial gain

If the publication is distributed under the terms of Article $25 \mathrm{fa}$ of the Dutch Copyright Act, indicated by the "Taverne" license above, 


\title{
The Agents-are-Substitutes Property in Continuous Generalized Assignment Problems
}

\author{
Reinder B. Lok *
}

\author{
Dolores Romero Morales ${ }^{\dagger}$
}

Dries Vermeulen ${ }^{\ddagger}$

March 18, 2005

\begin{abstract}
The VCG mechanism has some nice properties if the agents-are-substitutes property holds. For example, for combinatorial auctions the property assures that the VCG mechanism is supported by a pricing equilibrium. The existence of such a pricing equilibrium is a necessary condition for the existence of ascending auctions that are equivalent to the VCG mechanism. Although it is known that the agents-are-substitutes property is important in several settings few problems or subclasses of problems are proven to have the property. In this paper we show for a class of problems that the agents-are-substitutes property holds. Moreover we give two rather natural and small extensions that do not have this property in general. Furthermore we show that in our simple problem class we need the possibility of price discrimination.
\end{abstract}

\section{Introduction}

Classical optimization theory assumes that one planner has to find an optimal solution for a completely described problem. However in many practical situations knowledge and power may be distributed over different agents. These decentralized settings are an important issue in in supply chain management (SCM) where many producers and carriers work together to deliver the final product. From the field of mechanism design the VCG mechanism is a well known instrument to align the different goals of agents into a common goal by giving the right incentives. Although very nice in theory, the practical applicability of the VCG mechanism is limited. The main problem is that the mechanism might ask for a lot of computation and communication of confidential information. Many authors pointed out the importance of the so-called agents-are-substitutes property.

Ausubel and Milgrom [1] discuss why the agents-are-substitutes property is important for the single-item Vickrey auction. For combinatorial Vickrey auctions Bikhchandani et al. [3]

\footnotetext{
*Department of Quantitative Economics, Universiteit Maastricht, P.O. Box 616, 6200 MD Maastricht, The Netherlands; email: R.Lok@KE.unimaas.nl

${ }^{\dagger}$ Saïd Business School, University of Oxford, Park End Street, Oxford OX1 1HP, United Kingdom; email: Dolores.Romero-Morales@sbs.ox.ac.uk

${ }^{\ddagger}$ Department of Quantitative Economics, Universiteit Maastricht, P.O. Box 616, 6200 MD Maastricht, The Netherlands; email: D.Vermeulen@KE.unimaas.nl
} 
showed that the calculations for the VCG mechanism can be reduced considerably in case the agents-are-substitutes property holds. Furthermore, the property assures that their ascending price auction is equivalent to the VCG mechanism, i.e. the final transactions are the same. This is important because in these ascending auctions the communication of information is limited compared to the VCG mechanism itself. Other arguments in favor of ascending auctions are given by Cramton [6] and Kagel and Levin [9]. Bikhchandani et al. [3] conjecture that the agents-are-substitutes property is also a necessary condition for the equivalence of ascending auctions and the VCG mechanism. De Vries, Schummer and Vohra [16] prove that the stronger property of submodularity is in general necessary for the existence of an equivalent ascending auction.

Although it is known that the agents-are-substitutes property is important in several settings few problems or subclasses of problems are proven to have the property. Shapley [13] proved the agents-are-substitutes property for the classical assignment problem. Topkis [14] proved the stronger property of submodularity for the transportation problem. Bikhchandani et al. [3] show that the minimum spanning tree problem has the agents-are-substitutes property. They also show that the directed shortest path problem does not have the property. Gale and Politof [7] link substitutability with graph theory. For example they show that the assignment problem of Shapley [13] is a special case of the maximum weight circulation problem in a directed graph. Their main result is that substitutability corresponds to parallel arcs in the graph and complementarity corresponds to arcs in series.

Bikhchandani and Ostroy [4] show for the package assignment problem, which is a generalization of the classical assignment problem, that the agents-are-substitutes property holds if and only if the VCG mechanism corresponds to a pricing equilibrium and to a core allocation. In their problem formulation the equilibrium prices correspond to optimal dual variables. These results are a generalization of the results of Leonard [10] who discussed the classical assignment problem. Note that Bikhchandani and Ostroy [4] do not prove the agents-are-substitutes property itself for any class of problems. In this paper we prove the agents-are-substitutes property for a subclass of the package assignment problem. Essentially Bikhchandani and Ostroy [4] use general weakly increasing valuations where we restrict ourselves to concave piecewise linear valuations. As we actually prove the stronger property of submodularity, not only the results of Bikhchandani and Ostroy [4] but also the results of De Vries, Schummer and Vohra [16] apply to our problem class. We also show that the agents-are-substitutes property does not hold in general if valuations have a convex part due to setup-costs. Finally, the agents-are-substitutes property also does not hold in general if we restrict our setting to integral solutions.

The remainder of the paper is organized as follows. In section 2 we introduce the basic definitions of mechanism design. The subclass of the package assignment problem is described in section 3. Section 4 defines the agents-are-substitutes property, the related submodularity property, the pricing equilibrium and the core. Moreover, in this section we prove for our class of problems that the VCG mechanism needs price discrimination. The package assignment model itself is the subject of section 5 . We discuss the main results of Bikhchandani and Ostroy [4]. In section 6 we prove that our class of problems is submodular and therefore has the agents-are-substitutes property. In section 7 we present two extensions of the problem (which are still special cases of the package assignment problem) that do not have the agents-are-substitutes property. The paper ends with with a summary of our results 
and further research.

\section{Preliminaries}

\subsection{Mechanism design}

Game theory concerns the mathematical modeling and analysis of conflict situations between decision makers, called agents. For given situations it is analyzed how rational agents will act or interact. Mechanism design is a subfield of game theory. It is about the redesign of the conflict situation itself. By designing a mechanism for a conflict situation it is tried to align the individual goals of the agents into one common goal.

Before we give a formal definition of a mechanism we need the following. Let $A$ be a set of agents. Each agent $a \in A$ is characterized by its type, denoted by $t_{a}$. The set of all possible types that agent $a$ can possibly have is denoted by $T_{a}$ and is called its type space. Let $T \equiv \prod_{a \in A} T_{a}$ be the space of type profiles. Furthermore, let $O$ be a set of outcomes. Each agent $a$ has, associated with its type $t_{a}$, a preference relation $\succ_{t_{a}}$ on the set $O$ of outcomes. The preferences of the agents might be conflicting. Therefore the preferences of the agents have to be summarized in some way into a single preference relation on $O$. A function that maps every type profile on a single preference relation is called a social welfare function. A social choice function $o: T \rightarrow O$ is a function that chooses a most preferred outcome according to a social welfare function.

In mechanism design the goal is to implement the social choice function. This means that the outcome rule chooses the the most preferred outcome if the agents act rationally. Preferably the mechanism designer considers mechanisms in which agents have dominant actions, i.e. no matter the actions the other agents choose the dominant action is always the best to choose. In this way the mechanism designer can predict the actions of agents, and therefore the output that will be selected.

Assume that the agents' types are private information. In this setting it is not possible to implement the social choice function directly. Each agent $a \in A$ has a set $R_{a}$ of actions that it might possibly take. A mechanism chooses an output based on the actions the agents take. A mechanism is described by an outcome rule $\omega$ as well as payment functions $p_{a}$ for each $a \in A$. These are defined as follows. An outcome rule $\omega$ is a function from the set $R \equiv \prod_{a \in A} R_{a}$ to the set $O$ of outcomes. The payment function $p_{a}$ is a function from $R$ to $\mathbb{R}$ and represents the amount of money that agent $a$ has to pay. A mechanism in which the action of the agent is to report a type, i.e. $R_{a}=T_{a}$, is called a direct revelation mechanism.

For a given mechanism agents first choose their actions and after that an outcome is chosen and the agents pay the payments. Agents can influence the choice of the outcome by changing their actions. Moreover, these actions also influence the amount they have to pay.

In this paper an agent's preference given his type $t_{a}$ is assumed to be given by a valuation function $v_{a}\left(t_{a}, o\right)$ for outcomes $o \in O$. It is assumed that agents have quasi-linear utilities, i.e. $v_{a}\left(t_{a}, o\right)$ is in monetary units and the net valuation (or payoff) for outcome $o$ and payment $p$ is $v_{a}\left(t_{a}, o\right)-p$. Payments are a way to transfer utility from one agent to another agent. The utilities of the agents are Pareto optimal if no single agent can have more utility without 
decresing the utility of the other agents. By allowing payments it is assured that any Pareto optimal solution maximizes the total valuation of the agents. A social choice function that chooses an outcome that maximizes the total valuation is called a utilitarian social choice function.

\subsection{VCG mechanism}

A well known direct revelation mechanism that implements the utilitarian social choice function is the Vickrey auction. In this auction bidders bid on a single good, i.e. they report on their valuation for the good. The outcome is to sell the good to the bidder with the highest bid. That bidder has to pay the second highest bid. The dominant action for all agents is to bid their true valuation for the good. Therefore the good will be assigned to the agent with the highest valuation for it.

The Vickrey auction is generalized in the class of VCG mechanisms, named after Vickrey [15], Clarke [5] and Groves [8]. These mechanisms implement the utilitarian social choice function. Let $t=\left(t_{a}\right)_{a \in A}$ and $r=\left(r_{a}\right)_{a \in A}$ denote the true and reported preferences respectively. Furthermore let $v_{a}\left(t_{a}, o\right)$ and $v_{a}\left(r_{a}, o\right)$ be the true and reported valuations of agent $a$ for outcome $o$. The social choice function $\omega: T \rightarrow O$ chooses an outcome $o \in O$ that maximizes total welfare $\sum_{a \in A} v_{a}\left(t_{a}, o\right)$ given types $t$. This outcome is denoted by $o(t)$. The associated VCG mechanism chooses the outcome $o(r) \in \arg \max _{o \in O} \sum_{a \in A} v_{a}\left(r_{a}, o\right)$ when agents report $r$. So this mechanism mimics $\omega$ when the agents report truthfully. And in fact the VCG payments give the agents the incentive to report truthfully. Denote the maximal (reported) total welfare by $V(A, r) \equiv \sum_{a \in A} v_{a}\left(r_{a}, o(r)\right)$. The reported preferences of all agents but $a$ are denoted by $r^{-a}=\left(r_{b}\right)_{b \in A \backslash\{a\}}$. Finally let $h\left(r^{-a}\right)$ be an arbitrary function of the reported preferences of all agents but $a$. The class of VCG mechanisms is then characterized by the following payment by agent $a \in A$ :

$$
p_{a}(r) \equiv h\left(r^{-a}\right)-V(A, r)+v_{a}\left(r_{a}, o(r)\right) .
$$

The Vickrey auction [15] is a special case of the class of VCG mechanisms. For the Vickrey auction $h\left(r^{-a}\right)$ is equal to $V\left(A \backslash\{a\}, r^{-a}\right)$, i.e. the maximal reported welfare if agent $a$ is excluded from the auction. The net utility (or payoff) of agent $a$ is then

$$
v_{a}\left(t_{a}, o(r)\right)-\left[V\left(A \backslash\{a\}, r^{-a}\right)-V(A, r)+v_{a}\left(r_{a}, o(r)\right)\right] .
$$

This reduces to $V(A, t)-V\left(A \backslash\{a\}, t^{-a}\right)$ if $r=t$. This expression is also called the Vickrey payoff or the marginal contribution of agent $a$. In the following we assume $h\left(r^{-a}\right)=V(A \backslash$ $\{a\}, r^{-a}$ ) when we talk about the VCG mechanism.

The VCG mechanism has several nice properties, see Mas-Colell, Whinston and Green [11]. The VCG mechanism is truth revealing or incentive compatible (IC), which means that for any agent it is always best to report true preferences, no matter what the other agents do. Due to this property the mechanism is also called efficient, as the mechanism maximizes total reported welfare. Moreover, with the Vickrey payment function the mechanism is also individual rational (IR), i.e. an agent has a non-negative payoff as long as the dominant action is chosen.

In the next section we introduce a class of problems on which we apply the VCG mechanism. Thereafter we introduce the agents-are-substitutes property. This property is important 
for the practical use of the VCG mechanism. Therefore we show that for our class of problems the property holds.

\section{A class of decentralized planning problems}

\subsection{Introduction}

In this paper we consider a class of planning problems, namely continuous generalized assignment problems. A set of divisible goods has to be assigned to a set of agents. Each agent has a given capacity and each type of good has some per unit capacity consumption. We assume that the valuation of an agent is a linear function of the amount an agent gets of each good. Such planning problems have applications in transportation as well as in production planning. For example, consider a company that has to decide which freighters should transport its sales to the customers. The company has long term agreements with the freighters about the prices and available capacities. Due to the different locations of the freighters their costs, and therefore their profit margins, differ. Given that the company wants to maintain its long run relationship with the freighters, it is best to assign daily sales such that the total profit of the freighters is maximized given the capacities.

First we introduce the computationally easy mathematical problem. The problem becomes difficult when decision making is distributed over the agents. This will be discussed next. We assume that decentralized decision making emerges when the knowledge of parameters of the problem is distributed among agents.

\subsection{The model}

Let $A$ be the set of agents and $I$ the set of goods. Each agent $a \in A$ has a per-unit valuation $v_{a i}$ for each good $i \in I$, and a resource capacity $R_{a}$. Each good $i \in I$ requires an amount $r c_{i}$ of the resource for having one unit of that good. Without loss of generality all $R_{a}$ and $r c_{i}$ take on integer values. The goal of the planning problem is to assign as much of the goods as possible such that no capacity restrictions are violated and the total valuation is maximized. The planning problem can be written as

$$
\operatorname{maximize} \sum_{a \in A} \sum_{i \in I} v_{a i} x_{a i}
$$

subject to:

$$
\begin{aligned}
\sum_{i \in I} r c_{i} x_{a i} & \leq R_{a} \quad \forall a \in A \\
\sum_{a \in A} x_{a i} & \leq 1 \quad \forall i \in I \\
x_{a i} & \geq 0 \quad \forall a \in A, \forall i \in I
\end{aligned}
$$

where $x_{a i}$ is the fraction of good $i$ assigned to agent $a$. 
We will consider two special cases of (PP). First, the case in which $r c_{i}$ is equal to one for each $i$. This problem has the integrality property as the constraint matrix is totally unimodular and $R_{a}$ is integer. We call this the assignment problem with capacities (APC). Second, the subcase of (APC) that is also restricted to unit capacities, i.e. $R_{a}=1$ for each agent $a$, is the classical assignment problem (AP). The optimal objective value of a problem instance $\mathcal{I}$ of $(\mathrm{PP})$ is denoted by $V_{P P}(\mathcal{I})$. Furthermore, if $\mathcal{I}$ is also an instance of (APC) or (AP) we will write $V_{A P C}(\mathcal{I})$ and $V_{A P}(\mathcal{I})$ respectively instead of $V_{P P}(\mathcal{I})$ to emphasize the character of $\mathcal{I}$.

Problem (PP) is a model that does not have the integrality property in general. However for general (integer) $r c_{i}$ it is equivalent to a problem with the integrality property, i.e. it has the same objective value and there is a clear correspondence between the solutions: Consider a problem instance of (PP) with set of players $A$ and set of goods $I$. Good $i$ has an (integer) resource consumption equal to $r c_{i}$. We can split good $i$ into $r c_{i}$ goods with resource consumption equal to one. This set of goods is denoted by $I(i)$. The valuation of agent $a$ for good $\ell \in I(i)$ is equal to $v_{a \ell}=v_{a i} / r c_{i}$. Let $I^{\circ}=\bigcup_{i \in I} I(i)$ be the new set of goods. Then the planning problem is equivalent to a problem instance of (APC) with set of agents $A$, set of goods $I^{\circ}$ and valuations as given above. So any planning problem is equivalent to an assignment problem with capacities with the same set of agents. Due to this it is sufficient to prove the agents-are-substitutes property for (APC).

\subsection{Decentralization and the VCG mechanism}

We call (PP) the centralized problem. It can be solved by a single centralized planner in polynomial time. The problem becomes interesting when decision power is distributed over different agents. We assume that the valuations are privately known by the agents. In this decentralized setting a planner can only solve the planning problem to optimality after the agents report on their valuations. The planner has two problems. First, the planner has to acquire the information from the agents, so he should make it profitable for the agents to provide information. Second, the planner has to assure that the information given is correct. If the agents know that the planner will believe everything they say, they might have an opportunity to manipulate the planner's decision.

This is exactly the setting that can be handled with mechanisms as introduced in section 2. We will apply the VCG mechanism on the decentralized planning problem. The individual rationality property (IR) and the incentive compatibility property (IC) of the VCG mechanism solve the two problems of the planner. The output function of the VCG mechanism is to choose the optimal solution of (PP) with respect to the reported valuations, i.e. we assume that the planner treats the reports as if it is the truth. In the setting of (PP) the VCG mechanism has a third important property: it is (weakly) budget balanced, i.e. the sum of all the payments to the planner is non-negative. So the planner does not have to pay to solve its problems. It can easily be seen that even the individual payments in the planning problem are always positive, which is assured by (1) and (2).

Suppose we reformulate a planning problem as an assignment problem with capacities (APC). Then the integral decentralized problem is in fact very similar to a combinatorial auction. In these auctions the agents have private information on their valuations for combinations of goods and the auctioneer has to assign combinations of goods to the agents. The 
only difference with the combinatorial auction is that the bidders (agents) have a capacity. The combinatorial auction can be formulated as a set-packing problem which is an NP-hard problem, see for example De Vries and Vohra [17]. Moreover note that the size of the problem is exponential in the number of goods as agents should value each subset of goods. However the decentralized problem of (APC) is a polynomial problem as the valuation of the objects is linear and the linear programming relaxation has an integral solution.

\section{Important concepts in the VCG mechanism}

\subsection{Introduction}

The VCG mechanism has some drawbacks that are troublesome for its applicability. One drawback is that in many situations it is vulnerable to manipulation of groups of agents, see for example the discussion of the Vickrey auction by Ausubel and Milgrom [1]. Another drawback is that the mechanism needs the communication of a possibly exponential amount of information. For example, in combinatorial Vickrey auctions, agents have to value any subset of goods. Not only this communication complexity is problematic but in addition agents will prefer to communicate as little information as possible. The first drawback disappears in case the agents-are-substitutes property holds. The second drawback can be avoided or relieved if this property holds.

In the following sections we introduce the concepts that are important with respect to the VCG mechanism. First, we define the aforementioned agents-are-substitutes condition, and the related and slightly stronger concept of submodularity. Second we underpin the agentsare-substitutes property as a necessary condition for the existence of ascending price auctions. Related to this we introduce the definition of pricing equilibria. Moreover we show the need for the possibility of price discrimination. Thereafter we introduce the game theoretic core concept that is related to pricing equilibria.

\subsection{Substitutes and submodularity}

Following Shapley [13], Bikhchandani and Ostroy [4] defined the agents-are-substitutes property as follows. Let $R$ be a set and $V$ a function on all subsets of $R, V: 2^{R} \rightarrow \mathbb{R}$.

Definition 4.1 Elements in $R$ are called substitutes with respect to function $V$ if for all subsets $K \subseteq R$ :

$$
V(R)-V(K) \geq \sum_{a \in R \backslash K}[V(R)-V(R \backslash\{a\})] .
$$

In this paper we use the stronger property of submodularity. For completeness we show that agents are substitutes if $V$ is a submodular function. We start with the definition of submodularity which is also known as concavity in the field of game theory.

Definition 4.2 The function $V: 2^{R} \rightarrow \mathbb{R}$ is called a submodular function if for any two subsets 
$K$ and $L$ of $R$ the following inequality holds:

$$
V(K)+V(L) \geq V(K \cup L)+V(K \cap L) .
$$

An equivalent characterization of submodularity is given in the following, see for example Nemhauser and Wolsey [12].

Proposition 4.3 (Proposition 2.1 of Nemhauser and Wolsey [12, page 662]) The function $V: 2^{R} \rightarrow \mathbb{R}$ is a submodular function if and only if for any set $K \subseteq R$ and elements $a, b \notin K$ with $a \neq b$ the following holds:

$$
V(K \cup\{a\})-V(K) \geq V(K \cup\{a, b\})-V(K \cup\{b\}) .
$$

Now it can easily be seen that the agents-are-substitutes property is implied by submodularity.

Proposition 4.4 Consider the set function $V: 2^{R} \rightarrow \mathbb{R}$. If $V$ is a submodular function on the set $R$, then the elements of $R$ are substitutes with respect to $V$.

\section{Proof:}

We will prove that (4) holds for any $K \subseteq R$. This is clearly true for $K=R$, so consider an arbitrary $K \subset R$. Let $t=|R \backslash K|$ and $R \backslash K=\left\{a_{1}, \ldots, a_{t}\right\}$. Let $\tau$ be an arbitrary index in $\{1, \ldots, t\}$. By submodularity we know that

$$
V\left(R \backslash\left\{a_{1}, \ldots, a_{\tau-1}\right\}\right)-V\left(R \backslash\left\{a_{1}, \ldots, a_{\tau}\right\}\right) \geq V(R)-V\left(R \backslash\left\{a_{\tau}\right\}\right) .
$$

We can add up these inequalities for all $\tau$ in $\{1, \ldots, t\}$ :

$$
\sum_{\tau=1}^{t}\left[V\left(R \backslash\left\{a_{1}, \ldots, a_{\tau-1}\right\}\right)-V\left(R \backslash\left\{a_{1}, \ldots, a_{\tau}\right\}\right)\right] \geq \sum_{\tau=1}^{t}\left[V(R)-V\left(R \backslash\left\{a_{\tau}\right\}\right)\right] .
$$

This is equivalent to:

$$
V(R)-V(K) \geq \sum_{a \in R \backslash K}[V(R)-V(R \backslash\{a\})],
$$

and the desired result follows.

It is easy to show that if $|R| \leq 3$ submodularity is equivalent to the substitutes property.

\subsection{Ascending price auctions}

In some situations it is possible to replace the VCG mechanism with an ascending price auction and still get the same solution. An example of an ascending auction is the English auction. In the English auction bidders increase the price by outbidding each other until there is only one bidder left that wants to pay the price. It is well known that the English auction is equivalent to the Vickrey auction, see Vickrey [15], but has the advantage that most 
of the agents have to communicate much less information or less precise information than in the Vickrey auction itself. Bikhchandani et al. [3] give some other situations where such ascending pricing mechanisms can be used to imitate the VCG mechanism. They work with linear programming formulations in which dual variables correspond to prices and payoffs. Primal-dual algorithms correspond in some cases to ascending auctions. However the mechanism only converges to the VCG mechanism if the optimal dual variables (equilibrium prices) correspond to the VCG prices. This is assured if agents are substitutes, and Bikhchandani et al. [3] conjecture that this is also a necessary condition. De Vries, Schummer and Vohra [16] show that the slightly stronger property of submodularity is in general a necessary condition: They use a result of Ausubel and Milgrom [2] who proved for combinatorial auctions that submodularity (and hence the agents-are-substitutes property) is implied by the gross substitutes property of goods. Goods are called gross substitutes if the demand for one good is nondecreasing in the price of other goods. De Vries, Schummer and Vohra [16] argue that if the agents-are-substitutes property does not hold then the goods are no gross substitutes for at least one of the agents. They show that if goods are no substitutes for one agent, there exist gross substitutes valuation profiles for the other agents such that no ascending auction does yield the same transactions as the VCG mechanism.

Apart from the argument that ascending auctions need less communication, there is also some empirical evidence that ascending auctions are more useful than the VCG mechanism itself. In experimental tests of Kagel and Levin [9] it appears that in ascending auctions more players actually play the equilibrium strategy compared to the Vickrey auction. Players learn from the repetitive character of the auction and see less imaginary advantages from lying.

\subsection{The VCG mechanism is not supported by anonymous prices}

We start with the definition of a pricing equilibrium in the setting of (APC). Recall that the planning problem can be transformed into an assignment problem with capacities (APC) which has the integrality property. Let $x=\left(x_{a i}\right)_{a \in A, i \in I}$ be a feasible integral solution of (APC), and $X$ the set of all such solutions. Then $\left\{i \in I \mid x_{a i}=1\right\}$ is the set of goods that are assigned to agent $a$ by solution $x$. Furthermore, let $X(a)=\left\{x_{a} \mid x \in X\right\}$ be all possible values that $x_{a} \equiv\left(x_{a i}\right)_{i \in I}$ can have.

With this we can define $p_{a x_{a}}$ as the price that agent $a$ pays for getting the goods corresponding to $x_{a} \in X(a)$. Let $p=\left(p_{a x_{a}}\right)_{a \in A, x_{a} \in X(a)}$. These prices may depend on both the combination of goods corresponding to $x_{a}$ and the receiving agent. With such prices agent $a$ 's quasi-linear utility from receiving $x_{a}$ is equal to $u_{a x_{a}}(p) \equiv \sum_{i \in I} v_{a i} x_{a i}-p_{a x_{a}}$. Here quasilinear means that the utility is equal to the monetary valuation of the set of goods minus the amount paid for it. Suppose that the central planner of (APC) (called $s$ ) wants to maximize its revenue. Therefore, its net utility from allocating goods according to allocation $x \in X$ equals $u_{s x}(p) \equiv \sum_{a \in A} p_{a x_{a}}$, the total amount of money received.

Definition 4.5 A pricing equilibrium for (APC) is a combination of $x^{*} \in X$ and prices $p_{a x_{a}} \geq$ $0, a \in A$, such that

$$
\text { - } u_{a x_{a}^{*}}(p)=\max _{x_{a} \in X(a)}\left[\sum_{i \in I} v_{a i} x_{a i}-p_{a x_{a}}\right] \text { for all } a \in A,
$$


- $u_{s x^{*}}(p)=\max _{x \in X} \sum_{a \in A} p_{a x_{a}}$

Prices that do not discriminate between agents are called anonymous:

Definition 4.6 Prices $p$ are called anonymous if $p_{a x_{\circ}}=p_{b x_{\circ}}$ for all $a, b \in A$ and $x_{\circ} \in X(a) \cap$ $X(b)$.

The simplest anonymous prices are linear prices, i.e. prices that are just linear functions of the amount of goods:

Definition 4.7 Prices $p$ are called linear if for all $a \in A$ and $x_{a} \in X(a)$ we can write $p_{a x_{a}}$ as $\sum_{i \in I} \phi_{i} x_{a i}$. We call $\phi_{i}$ the per unit price of good $i$.

We now define a possible relationship between pricing equilibria and the VCG mechanism. Let $V_{A P C}(B, I)$ be the optimal objective value for an instance of (APC) with set of agents $B$ allocating set of goods $I$.

Definition 4.8 A pricing equilibrium $\left(x^{*}, p\right)$ is said to support the VCG mechanism if $u_{a x_{a}^{*}}(p)=$ $V_{A P C}(A, I)-V_{A P C}(A \backslash\{a\}, I)$ for all $a \in A$.

Leonard [10] showed for the assignment problem (AP) that the VCG mechanism is supported by some pricing equilibrium with linear prices. In section 5.4 we will see that there is a similar result for the assignment problem with capacities (APC). For (APC) the VCG mechanism is always supported by some pricing equilibrium, but here the prices are not necessarily linear. We now show for (APC) that we really need the possibility of non-anonymous prices for the VCG mechanism being supported by a pricing equilibrium.

Counterexample 4.9 Consider the following example of the planning problem. The set of agents $A$ consists of two agents $a$ and $b$ with capacities 1 and 2 respectively. The set of goods I consists of three goods $i, j$ and $k$ all of them with resource consumption 1 (i.e. $r c_{i}=r c_{j}=r c_{k}=1$ ). The agents' valuations for the goods are in Table 1. An optimal solution for this problem is

\begin{tabular}{cccc}
\hline & $i$ & $j$ & $k$ \\
\hline$a$ & 6 & 5 & 2 \\
$b$ & 5 & 4 & 1 \\
\hline
\end{tabular}

Table 1: Valuations for APC

that agent a gets good $k$, and agent $b$ gets goods $i$ and $j$. So the optimal value of the objective function is 11. To calculate the VCG-payoffs we also need the optimal values for the problems with one agent less. Obviously $V_{A P C}(\{a\})=6$ as a chooses good $i$ and $V_{A P C}(\{b\})=9$ as $b$ chooses goods $i$ and $j$. The VCG-payoffs are $\pi_{a}=V_{A P C}(\{a, b\})-V_{A P C}(\{b\})=11-9=2$, and $\pi_{b}=V_{A P C}(\{a, b\})-V_{A P C}(\{a\})=11-6=5$. For the optimal solution this means that agent $a$ pays 0 for good $k$ (which gives the utility $2-0=2$ ), and agent $b$ pays in total 4 for goods $i$ and $j$ (which gives the utility of $5+4-4=5$ ). The revenue for the planner is therefore $0+4=4$. With these values we get the following conflicting constraints for linear equilibrium prices: 
- The price of good $i$ should be at least 4 , otherwise agent a can improve its VCG-payoff by getting good $i$ and having payoff $6-\phi_{i}$.

- The price of good $j$ should be at least 3, otherwise agent a can improve its VCG-payoff by getting good $j$ and having payoff $5-\phi_{j}$.

- With these minimum prices, $b$ will have a net valuation for both goods $i$ and $j$ of 1 . Given its valuation for $k$ of 1 and given its capacity, agent $b$ can have a utility of at most 2, which is less than its VCG-payoff.

Note that allowing non-linear prices (i.e. prices for bundles of goods) does not give an equilibrium either. In that case we still have to define prices for single goods as the most simple type of bundles. We conclude that the VCG-mechanism is not supported, in general, by any pricing equilibrium with anonymous prices.

As said above, Leonard [10] showed for the assignment problem that the VCG mechanism is supported by some pricing equilibrium with linear prices. He showed that this pricing equilibrium can be found as a dual optimal solution of the assignment problem. Bikhchandani and Ostroy [4] showed a similar result with non-anonymous prices for the the so-called package assignment problem (PAP). Their result only holds if the agents-are-substitutes property holds. In section 5 we will see that (APC) is a special case of (PAP). This implies that the result of Bikhchandani and Ostroy [4] also holds for (APC).

\subsection{Core}

As proven above, the VCG mechanism may not be supported by an anonymous pricing equilibrium, i.e. it is not possible to announce goods prices such that each agent attains its VCG payoff. In the remainder of this paper it will become clear that the VCG mechanism for (APC) is supported by a general pricing equilibrium as described in definition 4.5. Bikhchandani and Ostroy [4] showed that there is a strong relation between such a pricing equilibrium and the the game theoretical concept of core. Before we present this concept, we give the definition of a transferable utility game (TU game).

Definition 4.10 A transferable utility game is a pair $(B, v)$ with set of players $B$ and characteristic function $v: 2^{B} \rightarrow \mathbb{R}$ with $v(\varnothing)=0$.

In the following we define the core of a TU game.

Definition 4.11 The core of the TU game $(B, v)$ is the set of all allocations $\theta \in \mathbb{R}^{B}$ that are feasible with respect the following restrictions:

$$
\begin{aligned}
\sum_{q \in K} \theta_{q} & \geq v(K) \forall K \subset B \\
\sum_{q \in B} \theta_{q} & =v(B) .
\end{aligned}
$$


From planning problem (PP) we define the following TU game. Consider the agents in the planning problem and the central planner, called $c$, as players of the game, so $B=A \cup\{c\}$. Let $v(K \cup\{c\})$ be equal to the objective value of (PP) if the agents in $K \subseteq A$ and the planner $c$ are available, which is denoted by $V_{P P}(K)$. So $v(K \cup\{c\})=V_{P P}(K)$. Let $v(K)$ be equal to zero for any set of players $K \subseteq A$.

One can see that a core allocation of the TU game associated with the planning problem is an allocation of the optimal objective value $V_{P P}(A, I)$ over the agents and the planner with a nice property, namely no subset of players (called coalition) has an incentive to act separately. In section 5.4 we will see that any core allocation implies a pricing equilibrium and any pricing equilibrium implies a core allocation. Moreover, the VCG mechanism corresponds to a core allocation in case the agents-are-substitutes condition is satisfied.

\section{Package assignment problem}

\subsection{Introduction}

The planning problem we introduced above is a special case of the package assignment problem of Bikhchandani and Ostroy [4]. Therefore its properties apply to the planning problem. In this section we will first introduce the package assignment problem. Second, we show that (PP) is a special case of it. Finally we discuss some properties of the package assignment problem that are proven by Bikhchandani and Ostroy [4]. They show that any core allocation implies a pricing equilibrium and any pricing equilibrium implies a core allocation. Moreover, the payoffs of the VCG mechanism correspond to a core allocation only if the agents-are-substitutes condition is satisfied.

\subsection{Model}

Bikhchandani and Ostroy [4] studied the package assignment problem (PAP). Their setting is an exchange economy where individuals trade packages of several kinds of indivisible goods. Individuals are divided into buyers and sellers. Each seller owns a number of goods while its valuation for ownership of goods is always zero. A buyer, on the other hand, does assign nonnegative value to owning (a set of) goods but has no initial endowment. Buyers are therefore willing to pay for the goods. The valuations are assumed to be weakly increasing in amounts per good. Sellers maximize the amount of money they receive while buyers maximize their net benefits from buying goods. Here it is assumed that the net benefits or utilities are quasi-linear, i.e. total utility is the sum of the monetary valuation for goods and the monetary transaction. Total welfare is therefore equal to the total monetary valuation of the buyers for the goods they acquire. The aim of the package assignment problem is to exchange packages of goods such that the total welfare over all buyers and sellers is maximized.

In the one seller setting, the package assignment problem reduces to a combinatorial auction. In this restricted setting the package assignment problem can be written as follows (see Bikhchandani et al. [3]). Let $B$ be a set of buyers and $M$ a set of goods to be sold by the seller indicated by $s$. Let $z=\left(z_{b}\right)_{b \in B}$ denote a partition of the goods where $z_{b}$ denotes the 
set of goods (or package) allocated to buyer $b$. The set of all possible partitions is denoted by $Z$. Let $y_{b S}$ be a binary variable that is equal to 1 if buyer $b$ gets set of goods $S \subseteq M$ and zero otherwise. The valuation of buyer $b$ for the set $S$ is $v_{b S} \geq 0$. Moreover let $Y_{z}$ be a binary variable that is equal to 1 if the seller chooses partition $z$ and zero otherwise. Then let $V_{P A P}(B)$ be the optimal objective value of the following winner determination problem with set of buyers $B$ :

$$
\text { maximize } \sum_{b \in B} \sum_{S \subseteq M} v_{b S} y_{b S}
$$

subject to:

$$
\begin{aligned}
\sum_{S \subseteq M} y_{b S} & \leq 1 \forall b \in B \\
\sum_{z \in Z} Y_{z} & \leq 1 \\
y_{b S} & \leq \sum_{z \in Z: z_{b}=S} Y_{z} \forall b \in B, \forall S \subseteq M \\
y_{b S} & \in\{0,1\} \forall b \in B, \forall S \subseteq M \\
Y_{z} & \in\{0,1\} \forall z \in Z .
\end{aligned}
$$

Constraints (5) impose that each buyer gets at most one package. Constraint (6) says that the seller chooses at most one partition of the goods. Note that the seller may restrict itself without loss of generality to partitions as valuations are nonnegative and buyers have no capacities. The third set of constraints (7) restricts buyers to the package that is offered by the seller. This restriction reflects an economy with no resale possibilities, which is called $\mathcal{E}_{3}$ in Bikhchandani and Ostroy [4]. If buyers can exchange the packages that are offered to them, (called economy $\mathcal{E}_{2}$ ) constraints (7) are replaced by the following constraint:

$$
\sum_{b \in B} y_{b S} \leq \sum_{z \in Z: z_{b}=S, b \in B} Y_{z} \quad \forall S \subseteq M .
$$

If buyers can exchange also individual objects (economy $\mathcal{E}_{1}$ ) the restriction reduces to the requirement that buyers receive non-overlapping sets of objects:

$$
\sum_{b \in B} \sum_{S \subseteq M: i \in S} y_{b S} \leq 1 \quad \forall i \in M .
$$

Effectively the choice $Y_{z}$ of the seller has become irrelevant in this case. For the case of multiple sellers Bikhchandani and Ostroy [4] also have a fourth economy $\mathcal{E}_{4}$. In this setting packages can also be distinguished by the seller. Economy $\mathcal{E}_{3}$ then reflects the case in which sellers can replace each other without approval of the buyer. Economy $\mathcal{E}_{4}$ forbids such a seller replacement. As we only consider the single seller case, we do not need the distinction between $\mathcal{E}_{3}$ and $\mathcal{E}_{4}$. Moreover, the main results of Bikhchandani and Ostroy [4] only apply to the single seller case.

From the optimization point of view (PAP) has some redundancy, there are two sets of variables that choose a partition: $Y$ for a preliminary allocation, and $y$ for a final allocation. The restrictions for the resale possibilities limit the difference between the two allocations. However, note that any type of resale restrictions allows for all possible partitions of the 
goods. The reason to model the underlying optimization problem as an exchange economy is that the dual variables of constraints (7), (8) and (9) can be interpreted as prices of addressed packages, anonymous packages and anonymous goods respectively. These are the types of prices that may exist in economies $\mathcal{E}_{3}, \mathcal{E}_{2}$ and $\mathcal{E}_{1}$ respectively. As we proved in section 4.4 that anonymous prices are not sufficient to support the VCG mechanism for the decentralized planning problem, we will only consider economy $\mathcal{E}_{3}$.

\subsection{Special case}

Our decentralized planning problem is very similar to the package assignment problem. In section 3.2 we showed that any instance of the continuous planning problem is equivalent to an instance of (APC) that has the integrality property. We may therefore restrict ourselves to problems with $r c_{i}=1$ that have the integrality property. This also means that for finding an optimal planning, we can restrict ourselves to integral solutions. Therefore the task of the planner is to assign each good to one agent. This is the same as the task of the seller in (PAP).

There are two important differences between our planning problem and the package assignment problem. On one hand the planning problem is easier as it deals with linear valuations instead of general weakly increasing valuation functions. On the other hand it is more difficult because of the capacities the agents face. This means that both sides of the economy have capacity restrictions. However, the capacities can easily be implemented in the package assignment setting by assuming disposability. This means that an agent's valuation of a set of goods is equal to the highest valued subset that fulfills its capacity constraint. We may observe that these valuations are weakly increasing. In this way the capacity constraints are implemented in the valuation function, and the planning problem appears to be a special case of the package assignment model. Therefore, all the results shown for the package assignment model by Bikhchandani and Ostroy [4] also hold for our planning problem. In section 5.4 we will present the results that are relevant for our discussion.

\subsection{Properties}

As already said, in this paper we will focus on the single seller case of the package assignment problem, even though some of the results of Bikhchandani and Ostroy [4] are also true for multiple sellers. Problem (PAP) is a generalization of the classical assignment problem. Leonard [10] proved for the classical assignment problem that a dual optimal solution corresponds both to a linear pricing equilibrium and the VCG solution. The results of Bikhchandani and Ostroy [4] are a generalization of the work of Leonard. The first result for the package assignment problem is about pricing equilibria.

Bikhchandani and Ostroy [4] proved that a pricing equilibrium (as defined in section 4.4) corresponds to an integral optimal solution of the linear program (PAP).

Theorem 5.1 (Theorem 3.1 of Bikhchandani and Ostroy [4]) A combination of a solution for (PAP) and prices $p_{b S}$ for all buyers $b \in B$ and all $S \subseteq M$ is a pricing equilibrium if and only if the solution is optimal for (PAP) and the combination of the prices and the payoffs of the buyers and seller constitute a dual optimal solution of the LP-relaxation of (PAP). 
From this theorem one can conclude that pricing equilibria are efficient, and have a one-toone correspondence with dual optimal optimal solutions of (PAP). The following theorem also gives a one-to-one correspondence between core allocations and pricing equilibria. Similar as we defined a TU game for (PP) in section 4.5, we may define a TU game for the package assignment model.

Theorem 5.2 (Theorem 5.1 of Bikhchandani and Ostroy [4]) A combination of a solution for (PAP) and prices $p_{b S}$ for all buyers $b \in B$ and all $S \subseteq M$ is a pricing equilibrium if and only if the corresponding payoffs of the buyers and seller constitute a core allocation of $V_{P A P}(B)$ over $B \cup\{s\}$.

So combining the two theorems, there is a one-to-one correspondence between pricing equilibria and core allocations. Moreover Bikhchandani and Ostroy [4] show that the core is nonempty: giving the seller a payoff of $V_{P A P}(B)$ and all the buyers a payoff of zero fulfills all core constraints.

The substitutes property of the buyers is the necessary and sufficient condition for which the VCG mechanism leads to both a core allocation of the total utilities and a certain pricing equilibrium.

Theorem 5.3 (Theorem 6.1 of Bikhchandani and Ostroy [4]) The following statements are equivalent for (PAP):

(i) Buyers are substitutes.

(ii) The payoff vector of the VCG mechanism is a core allocation of $V_{P A P}(B)$ over $B \cup\{s\}$.

(iii) The VCG mechanism corresponds to the pricing equilibrium in $\mathcal{E}_{3}$ with prices that are the lowest possible equilibrium prices for all buyers.

As pricing equilibria correspond to optimal solutions of the dual linear programming problem of (PAP), statement (iii) of Theorem 5.3 also implies that the VCG mechanism corresponds to an optimal dual solution that minimizes the payments of the buyers. An important implication of this is that all VCG-payments and prices can be calculated in two steps. First we calculate the optimal solution for (PAP). Second, we find the optimal dual solution that minimizes the prices of the bidders. In this way we get the VCG payoffs by solving only two linear programming problems. Note that this considerably lowers the computational burden as by using the definition of VCG payments we would have to calculate $V_{P A P}(B \backslash\{b\})$ for all $b \in B$.

\section{The substitutes property for the planning problem}

\subsection{Introduction}

In the previous sections we saw that the agents-are-substitutes property is important with respect to the possible implementation of the VCG mechanism. In this section we prove that the planning problem has the agents-are-substitutes property. In fact we prove the stronger 
property of submodularity. The proof is done in three steps. First we prove that the classical assignment problem is submodular. This is a generalization of a result of Shapley [13] who proved the agents-are-substitutes property. Second we generalize the submodularity property to the assignment problem with capacities (APC). Finally the submodularity of the planning problem is an easy consequence. In section 6.4 it is shown that the planning problem retains the submodularity property when the valuations become concave piecewise linear due to additional capacities.

\subsection{The assignment problem is submodular}

In this section we consider the planning problem where the capacities $R_{a}$ and the resource consumption $r c_{i}$ are equal to one. As said before this is the classical assignment problem (AP). In this section we prove that the assignment problem is submodular with respect to the set of agents. In the following we will consider related instances of the assignment problem. In order to discriminate between these problem instances we use the notation $A P(A, I)$ which indicates an instance of the assignment problem with agent set $A$ and set of goods $I$. The optimal solution value of $A P(A, I)$ is denoted by $V_{A P}(A, I)$.

Theorem 6.1 The function $V_{A P}(\cdot, I): 2^{A} \rightarrow \mathbb{R}$ is submodular in $A$.

\section{Proof:}

It is enough to prove that the conditions in Proposition 4.3 are fulfilled. The proof is by induction on the cardinality of the set of agents $A$. We may observe that the conditions of Proposition 4.3 only make sense when $|A| \geq 2$.

Suppose $A=\{a, b\}$ and $K=\varnothing$. Obviously $V_{A P}(\varnothing, I)=0$. Moreover we know that $V_{A P}(\{a\}, I)+V_{A P}(\{b\}, I) \geq V_{A P}(\{a, b\}, I)$ because of optimality of the left hand side. Combining these observations, we get

$$
V_{A P}(\{a\}, I)-V_{A P}(\varnothing, I) \geq V_{A P}(\{a, b\}, I)-V_{A P}(\{b\}, I) .
$$

As we chose $a$ and $b$ arbitrarily, the function $V_{A P}(\cdot, I)$ is submodular for $|A|=2$.

Suppose that the conditions in Proposition 4.3 are fulfilled for $|A|=k, k \geq 2$. Then we want to show the submudularity result for $|A|=k+1$. Let $K \subseteq A$ be a set of $k-1$ agents and let $a$ and $b$ be two arbitrary agents not in $K, a \neq b$. First we prove that the marginal value of an additional good will not decrease if we add an arbitrary agent to an instance of the assignment problem with $k-1$ agents, i.e. for $i \in I$ we have:

$$
V_{A P}(K, I)-V_{A P}(K, I \backslash\{i\}) \leq V_{A P}(K \cup\{b\}, I)-V_{A P}(K \cup\{b\}, I \backslash\{i\}) .
$$

Consider the instance $A P(K, I)$ of the assignment problem and suppose that it is optimal to assign good $i$ to agent $c$. Then:

$$
\begin{aligned}
V_{A P}(K, I)- & V_{A P}(K, I \backslash\{i\}) \\
& =v_{c i}+V_{A P}(K \backslash\{c\}, I \backslash\{i\})-V_{A P}(K, I \backslash\{i\}) \\
& \leq v_{c i}+V_{A P}((K \cup\{b\}) \backslash\{c\}, I \backslash\{i\})-V_{A P}(K \cup\{b\}, I \backslash\{i\}) \\
& \leq V_{A P}(K \cup\{b\}, I)-V_{A P}(K \cup\{b\}, I \backslash\{i\}) .
\end{aligned}
$$


The equality holds by the definition of good $i$. The first inequality is due to the induction hypothesis. The final inequality holds because $V_{A P}(K \cup\{b\}, I)$ is the value of an optimal assignment. If $i$ is not assigned to any agent, then expression (10) holds trivially as the left hand side will equal zero.

Now we are able to show the submodularity result when $|A|=k+1$. Consider the problem instance $A P(K \cup\{a, b\}, I)$, and suppose that it is optimal to assign good $j$ to agent $a$. Then we have:

$$
\begin{aligned}
V_{A P}(K \cup\{a, b\}, I)-V_{A P}(K \cup\{b\}, I) \\
\quad=v_{a j}+V_{A P}(K \cup\{b\}, I \backslash\{j\})-V_{A P}(K \cup\{b\}, I) \\
\leq v_{a j}+V_{A P}(K, I \backslash\{j\})-V_{A P}(K, I) \\
\leq V_{A P}(K \cup\{a\}, I)-V_{A P}(K, I) .
\end{aligned}
$$

The first inequality holds because of (10). The second inequality holds because $V_{A P}(K \cup$ $\{a\}, I)$ is the optimal value of $A P(K \cup\{a\}, I)$. So we found the following:

$$
V_{A P}(K \cup\{a\}, I)-V_{A P}(K, I) \geq V_{A P}(K \cup\{a, b\}, I)-V_{A P}(K \cup\{b\}, I) .
$$

If there is no good assigned to agent $a$ this inequality still holds as the right hand side will be equal zero, and thus the desired result follows.

The theorem above is an extension of the result of Shapley [13] who proved that the assignment problem had the substitutes property with respect to the agents. According to Proposition 4.4, the result of Shapley follows from Theorem 6.1.

\subsection{The assignment problem with capacities is submodular}

In this section we analyze the assignment problem with capacities (APC), i.e. consumptions $r c_{i}$ are equal to one, like in the assignment problem, but capacities are arbitrary integers. We will show that this problem is still submodular. Let $A P C(A, I)$ denote a problem instance of (APC) with set of agents $A$ with resource capacities $\left(R_{a}\right)_{a \in A}$, and set of goods $I$. The optimal value of $A P C(A, I)$ is denoted by $V_{A P C}(A, I)$.

Theorem 6.2 The function $V_{A P C}(\cdot, I): 2^{A} \rightarrow \mathbb{R}$ is submodular in $A$.

\section{Proof:}

In order to show that the problem is submodular we construct an equivalent classical assignment problem. For each $a \in A$, we split agent $a$ into $R_{a}$ agents, say $a_{1}, \ldots, a_{R_{a}}$, with resource capacity 1 and the same valuations as agent $a$. Let $A^{\circ}$ denote the new set of agents. It is easy to see that the assignment problem $A P\left(A^{\circ}, I\right)$ has the same objective value as $A P C(A, I)$. From Theorem 6.1 we know that $A P\left(A^{\circ}, I\right)$ is submodular with respect to the set of agents $A^{\circ}$. Let $K \subseteq A$ and $K^{\circ}$ be the corresponding agents in $A^{\circ}$. For $a, b \notin K$ with $a \neq b$ we have that

$$
\begin{aligned}
V_{A P C}(K \cup\{a\}, I)-V_{A P C}(K, I) \\
=V_{A P}\left(K^{\circ} \cup\left\{a_{1}, \ldots, a_{R_{a}}\right\}, I\right)-V_{A P}\left(K^{\circ}, I\right)
\end{aligned}
$$




$$
\begin{aligned}
& \geq V_{A P}\left(K^{\circ} \cup\left\{a_{1}, \ldots, a_{R_{a}}\right\} \cup\left\{b_{1}\right\}, I\right)-V_{A P}\left(K^{\circ} \cup\left\{b_{1}\right\}, I\right) \\
& \geq \\
& \vdots \\
& \geq V_{A P}\left(K^{\circ} \cup\left\{a_{1}, \ldots, a_{R_{a}}\right\} \cup\left\{b_{1}, \ldots, b_{R_{b}}\right\}, I\right)-V_{A P}\left(K^{\circ} \cup\left\{b_{1}, \ldots, b_{R_{b}}\right\}, I\right) \\
& =V_{A P C}(K \cup\{a, b\}, I)-V_{A P C}(K \cup\{b\}, I),
\end{aligned}
$$

in which the inequalities follow by the submodularity of the assignment problem, and the desired result holds.

Topkis [14] has proved that the transportation problem is a submodular function of the agents' capacities. The transportation problem is an equivalent formulation to (APC) in terms of cost minimization. The proof above is shorter and more intuitive.

Recall from section 3 that any planning problem is equivalent to an instance of (APC). Let $\operatorname{PP}(A, I)$ be an instance of the planning problem with set of agents $A$ and set of goods $I$. Let $I^{\circ}$ be the new set of goods that emerges by splitting the goods of $I$. Then we know that $V_{P P}(A, I)=V_{A P C}\left(A, I^{\circ}\right)$. As $V_{A P C}\left(A, I^{\circ}\right)$ is submodular in $A$, we have the following corollary.

Corollary 6.3 The function $V_{P P}(\cdot, I): 2^{A} \rightarrow \mathbb{R}$ is submodular in $A$.

\subsection{Concave piecewise linear valuations}

In this section we discuss a generalization of the planning problem that still has the substitutes property with respect to the agents. Assume that agents can hire limited amounts of additional resource capacity, say human resources or overtime. Let $R_{a}^{e}$ be the maximum overtime that agent $a$ can hire for wage $w^{e}$ per unit. Furthermore let $E$ be the set of possible overtime wages. As an agent will first use its cheapest resource capacity, the problem corresponds to concave piecewise linear valuation functions. Consider the following model,

$$
\operatorname{maximize} \sum_{a \in A} \sum_{i \in I} v_{a i} x_{a i}-\sum_{e \in E} \sum_{a \in A} w^{e} o_{a}^{e}
$$

subject to:

$$
\begin{aligned}
\sum_{i \in I} r c_{i} x_{a i} & \leq R_{a}^{\text {fixed }}+\sum_{e \in E} o_{a}^{e} \quad \forall a \in A \\
o_{a}^{e} & \leq R_{a}^{e} \quad \forall a \in A, \forall e \in E \\
\sum_{a \in A} x_{a i} & \leq 1 \quad \forall i \in I \\
x_{a i} & \geq 0 \quad \forall a \in A, \forall i \in I \\
o_{a}^{e} & \geq 0 \quad \forall a \in A, \forall e \in E
\end{aligned}
$$

where $o_{a}^{e}$ is the overtime of wage $w^{e}$ hired by agent $a$. In this problem $R_{a}^{f i x e d}$ is the fixed (normal) capacity of agent $a$. 
We show that the optimal value of the objective function of (PPO) is still a submodular function of the set of agents. We do this by reformulating it as a planning problem with linear valuations. Let $\operatorname{PPO}(A, I)$ denote a problem instance of the planning problem with overtime with set of agents $A$ and set of goods $I$. Denote the optimal value of $\operatorname{PPO}(A, I)$ by $V_{P P O}(A, I)$. Agent $a$ can be split into $1+|E|$ agents with capacities $R_{a}^{f}$ and $R_{a}^{e}, e \in E$ respectively. The first new agent has valuations $v_{a i}$ that correspond to the use of the fixed capacity, and the other new agents have valuations $v_{a i}-w^{e} r c_{i}$ that correspond to using the overtime of wage $w^{e}, e \in E$ with rate $r c_{i}$. Let $A^{\circ}$ be the new set of agents with the valuations and capacities as given above. Then $V_{P P O}(A, I)=V_{P P}\left(A^{\circ}, I\right)$. Similarly to the proof of Theorem 6.2 we get the following corollary.

Corollary 6.4 The function $V_{P P O}(\cdot, I): 2^{A} \rightarrow \mathbb{R}$ is submodular in $A$.

\section{Impossibilities}

\subsection{Introduction}

In section 6 we proved that the planning problem is submodular in the set of agents, and therefore has the agents-are-substitutes property according to Proposition 4.4. Moreover for a generalization with concave piecewise linear valuations the problem retains this property. Unfortunately the agents-are-substitutes property gets lost for other rather natural and small extensions of the planning problem. So far we assumed that the goods are divisible. For example, the transport of oil can easily be divided over different carriers. On the contrary, many goods are indivisible. For example, you can not split a cow over different cattle-trucks. We will therefore analyze the planning problem when goods are indivisible in section 7.2. Another extension of the planning problem arises from its application in production planning. In many real-life settings production capacity is only available after the production line has been set up. For this we take setup costs into account in section 7.3.

\subsection{Generalized assignment problem}

We have seen that when the resource consumptions $r c_{i}$ are equal to one, we can assume without loss of optimality that the variables $x_{a i}$ are binary. In this section we study the planning problem where each good should be assigned to exactly one agent, i.e. problem (PP) where the nonnegativity constraints (3) on $x_{a i}$ are replaced by

$$
x_{a i} \in\{0,1\} \quad \forall a \in A, \forall i \in I .
$$

This problem is also known as the generalized assignment problem (GAP). An instance of the problem is denoted by $G A P(A, I)$ with $A$ the set of agents and $I$ the set of goods. The optimal objective function of such an instance is denoted by $V_{G A P}(A, I)$.

Again, the substitutes property does not hold in general. Consider the following problem instance. We have three agents, $a, b$ and $c$ with capacities 2, 2 and 1 respectively, and three 
goods, $i, j$ and $k$ with resource consumption 2, 1 and 1 respectively. The agents' valuations for the goods are given in Table 2.

\begin{tabular}{cccc}
\hline & $i$ & $j$ & $k$ \\
\hline$a$ & 6 & 5 & 5 \\
$b$ & 0 & 0 & 3 \\
$c$ & 0 & 3 & 0 \\
\hline
\end{tabular}

Table 2: Valuations for GAP

Consider problem instances $G A P(K, I)$ for the following $K \subseteq A$.

- For $K=\{a, b, c\}$ we have $V_{G A P}(K, I)=12$ :

Agent $a$ gets good $i$,

Agent $b$ gets good $k$,

Agent $c$ gets good $j$.

- For $K=\{a, c\}$ we have $V_{G A P}(K, I)=10$ :

Agent $a$ gets goods $j$ and $k$.

- For $K=\{a, b\}$ we have $V_{G A P}(K, I)=10$ :

Agent $a$ gets goods $j$ and $k$.

Agent $b$ gets good $i$.

- For $K=\{a\}$ we have $V_{G A P}(K, I)=10$ :

Agent $a$ gets goods $j$ and $k$.

Now the following substitutes condition is violated, with $V(\cdot) \equiv V_{G A P}(\cdot, I)$ :

$$
V(\{a, b, c\})-V(\{a\}) \geq V(\{a, b, c\})-V(\{a, c\})+V(\{a, b, c\})-V(\{a, b\})
$$

as well as the following submodularity condition

$$
V(\{a, c\})-V(\{a\}) \geq V(\{a, b, c\})-V(\{a, b\}) .
$$

\subsection{Setup costs}

The planning problem assumes a linear valuation function for the agents. In this section we include a setup costs $f_{a}$ in the valuation function, i.e.,

$$
\text { maximize } \sum_{a \in A} \sum_{i \in I} v_{a i} x_{a i}-\sum_{a \in A} f_{a} y_{a}
$$

subject to:

$$
\begin{aligned}
\sum_{i \in I} r c_{i} x_{a i} & \leq R_{a} y_{a} \quad \forall a \in A \\
\sum_{a \in A} x_{a i} & \leq 1 \quad \forall i \in I \\
x_{a i} & \geq 0 \quad \forall a \in A, \forall i \in I \\
y_{a} & \in\{0,1\} \quad \forall a \in A
\end{aligned}
$$


where $x_{a i}$ is defined as before and $y_{a}$ is equal to one if agent $a$ (partially) processes at least one good. An instance of the problem is denoted by $\operatorname{PPS}(A, I)$ with $A$ the set of agents and $I$ the set of goods. The optimal objective function of such an instance is denoted by $V_{P P S}(A, I)$. We show by giving a counterexample that (PPS) does not enjoy the agents-aresubstitutes property. Consider the following problem instance. We have three agents, $a, b$ and $c$ all three with capacity 2, and setup costs of 10,1 and 1 respectively. There are four goods, $i, j, k$ and $m$, all with resource consumption equal to 1 . The agents' valuations for the goods are given in Table 3.

\begin{tabular}{ccccc}
\hline & $i$ & $j$ & $k$ & $m$ \\
\hline$a$ & 4 & 4 & 6 & 6 \\
$b$ & 1 & 1 & 2 & 1 \\
$c$ & 1 & 1 & 1 & 10 \\
\hline
\end{tabular}

Table 3: Valuations for PPS

Consider problem instances $P P S(K, I)$ for the following $K \subseteq A$.

- For $K=\{a, b, c\}$ we have $V_{P P S}(K, I)=12$ :

Agent $a$ gets no goods,

Agent $b$ gets goods $i$ and $k$,

Agent $c$ gets goods $j$ and $m$.

- For $K=\{a, c\}$ we have $V_{P P S}(K, I)=10$ :

Agent $a$ gets goods $i$ and $k$,

Agent $c$ gets goods $j$ and $m$.

- For $K=\{a, b\}$ we have $V_{P P S}(K, I)=3$ :

Agent $a$ gets good $k$ and $m$,

Agent $b$ gets good $i$ and $j$.

- For $K=\{a\}$ we have $V_{P P S}(K, I)=2$ :

Agent $a$ gets good $k$ and $m$.

Now the following substitutes condition is violated, with $V(\cdot) \equiv V_{P P S}(\cdot, I)$ :

$$
V(\{a, b, c\})-V(\{a\}) \geq V(\{a, b, c\})-V(\{a, c\})+V(\{a, b, c\})-V(\{a, b\}),
$$

as well as the submodularity condition

$$
V(\{a, c\})-V(\{a\}) \geq V(\{a, b, c\})-V(\{a, b\}) .
$$

Problem (PPS) can be formulated as a special case of (PAP) for which weakly increasing valuations are assumed. The setup costs cause downward jumps in the valuations of the agents. By assuming disposability we can set the negative part of these functions equal to zero. Observe that the optimal solution to this problem is not necessarily a partition of the goods. 


\section{Conclusions and further research}

In this paper we introduced a class of planning problems where the decision power is distributed over the agents. The VCG mechanism is a well known instrument to deal with those decentralized settings, but it may be impractical. Many authors pointed out the importance of the agents-are-substitutes property for the practical applicability of the VCG mechanism. Although it is known that the property is important in several settings few problems or subclasses of problems are proven to have the property. We proved for our planning problem that the agents-are-substitutes property holds, and that even the stronger property of submodularity holds. Moreover we showed that by introducing setup costs, which makes the valuations partly convex, the agents-are-substitutes property does not hold in general. The same is true for the integer version of our problem class, which is known as the generalized assignment problem.

The importance of the agents-are-substitutes property and the submodularity property is respectively shown by Bikhchandani and Ostroy [4], and De Vries, Schummer and Vohra [16] and Bikhchandani et al. [3]. First, our planning problem can be reformulated as a special case of the package assignment problem introduced by Bikhchandani and Ostroy [4]. In this problem the agents-are substitutes property is a necessary and sufficient condition for the VCG mechanism to correspond to a pricing equilibrium. We showed that we can not restrict ourselves to linear pricing equilibria as in some cases price discrimination is needed. Second, due to the results of De Vries, Schummer and Vohra [16] and Bikhchandani et al. [3] submodularity implies that there exists an ascending auction that implements the VCG mechanism.

The class of problems we encountered in this paper is inspired by supply chain planning. We gave an example of a company that has to decide which freighters should transport its sales to the customers. The company wants to maximize the total profit of the freighters by assigning daily sales appropriately. In this example the actions of the freighters are aligned by the VCG mechanism. All these freighters are part of one level in a supply chain, and are

proven to be substitutes. In the future we want to investigate the applicability of mechanism design to settings in which the different suppliers and buyers in a supply chain are the agents whose actions should be aligned to improve supply chain performance. In this setting a buyer and its supplier are each others complements. Therefore the VCG mechanism does not seem to be applicable.

\section{References}

[1] Lawrence M. Ausubel and Paul Milgrom. The lovely but lonely Vickrey auction. In Peter Cramton, Yoav Shoham, and Richard Steinberg, editors, Combinatorial Auctions, chapter 1. MIT Press, 2005. Forthcoming.

[2] Lawrence M. Ausubel and Paul R. Milgrom. Ascending auctions with package bidding. Frontiers of Theoretical Economics, 1(1):1-42, 2002.

[3] Sushil Bikhchandani, Sven de Vries, James Schummer, and Rakesh V. Vohra. Linear programming and Vickrey auctions. In Brenda Dietrich and Rakesh V. Vohra, editors, 
Mathematics of the Internet - E-Auction and Markets, pages 75-116. Springer-Verlag, Berlin, 2002.

[4] Sushil Bikhchandani and Joseph M. Ostroy. The package assignment model. Journal of Economic Theory, 107(2):377-406, December 2002.

[5] Edward H. Clarke. Multipart pricing of public goods. Public Choice, 11:17-33, 1971.

[6] Peter Cramton. Ascending auctions. European Economic Review, 42:745-756, 1998.

[7] David Gale and Themistocles Politof. Substitutes and complements in network flow problems. Discrete Applied Mathematics, 3:175-186, 1981.

[8] Theodore Groves. Incentives in teams. Econometrica, 41(4):617-631, July 1973.

[9] John H. Kagel and Dan Levin. Behavior in multi-unit demand auctions: experiments with uniform price and dynamic Vickrey auctions. Econometrica, 69(2):413-454, March 2001.

[10] Herman B. Leonard. Elicitation of honest preferences for the assignment of individuals to positions. Journal of Political Economy, 91(3):461-479, 1983.

[11] Andreu Mas-Colell, Michael D. Whinston, and Jerry R. Green. Microeconomic Theory. Oxford University Press, New York, 1995.

[12] George L. Nemhauser and Laurence A. Wolsey. Integer and Combinatorial Optimization. John Wiley \& Sons, New York, 1988.

[13] Lloyd S. Shapley. Complements and substitutes in the optimal assignment problem. Naval Research Logistics Quarterly, 9:45-48, 1962.

[14] Donald M. Topkis. Supermodularity and Complementarity. Frontiers of Economic Research. Princeton University Press, Princeton, New Jersey, 1998.

[15] William Vickrey. Counterspeculation, auctions, and competitive sealed tenders. The Journal of Finance, 16(1):8-37, March 1961.

[16] Sven de Vries, James Schummer, and Rakesh V. Vohra. On ascending Vickrey auctions for heterogeneous objects. Work in progress, November 2003.

[17] Sven de Vries and Rakesh V. Vohra. Combinatorial auctions: A survey. INFORMS Journal on Computing, 15(3):284-309, 2003. 